\title{
A BIOLOGICAL VIEW ON INFORMATION ECOSYSTEMS
}

\author{
BENGT CARLSSON AND PAUL DAVIDSSON \\ Department of Software Engineering and Computer Science \\ Blekinge Institute of Technology, 37125 Ronneby, Sweden \\ E-mail: bengt.carlsson@bth.se,paul.davidsson@bth.se
}

\begin{abstract}
We study the dynamics of information ecosystems where there are antagonistic agents or groups of antagonistic agents. In particular, we focus on systems that consist of exploiter agents and agents being exploited. When comparing information ecosystems with biological ecosystems, it becomes clear that both types of systems seem to support robust solutions that are hard to violate by a single agent. In the analysis of information ecosystems, it is important to take into consideration that agents may have a Machiavellian intelligence, i.e., that they take the self-interest of other agents into consideration. We conclude that in the interaction between antagonistic agents within information systems, arms race is a major force. A positive result of this is a better preparedness for innocent agents against the vigilant agents. Some examples are given to show how the modelling of information ecosystems in this way can explain the origin of more robust systems when antagonistic agents are around.
\end{abstract}

\section{Introduction}

The emerging global information infrastructure may be regarded as a universal information ecosystem. An information ecosystem is populated by infohabitants, i.e., (semi-) autonomous software entities typically acting on the behalf of humans. These infohabitants, or agents, may or may not have conflicting interests. In this paper we will compare the dynamic process resulting from the interaction between agents in information ecosystems that have conflicting interests to the processes within biological ecosystems.

Biological ecosystems are basically determined by an individually centered mechanism called natural selection $[9,10]$. Normally, fitness expresses the rate of success for individuals within an ecosystem. If antagonistic agents of an information ecosystem that are acting in their own interests, are modeled as part of an independent biological system, this will represent a worst-case scenario because no cooperation can be taken for granted. As a result, the robustness of an information ecosystem may be explained from a truly distributed point of view.

In section 2 we will examine the interaction between agents and between agents and the humans behind. Section 3 provides some Internet-based examples of antagonistic interactions, and is followed by a discussion of the dynamics of the information ecosystem compared to a biological system. Finally, in section 4 some conclusions are drawn. 


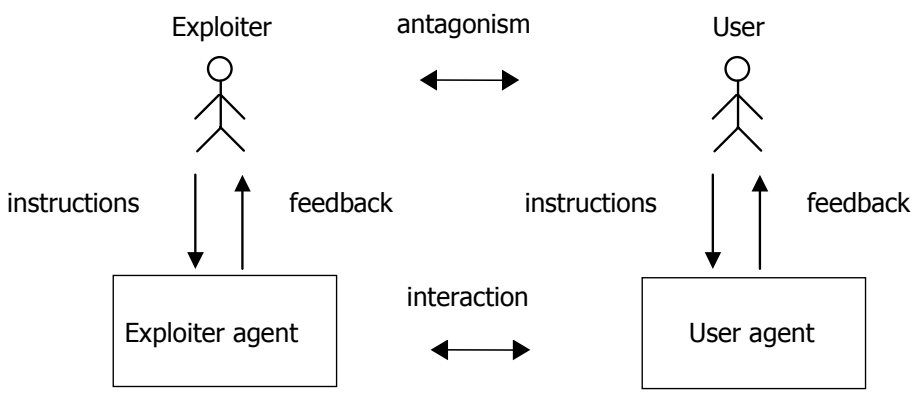

Fig. 1. Part of an antagonistic information ecosystem.

\section{The dynamics of antagonistic information ecosystems}

Humans have the possibility to represent knowledge outside the brain as mind-tools $[2,3]$. Computers, "intelligent" systems and agent technology within the global network may all be regarded as mind-tools, controlled by independent and selfish humans.

In his book "The Prince", Machiavelli wrote about how to conquer and preserve authority. The impression of being fair and honest may, if the preservation of the authority requires it, be followed by tricks, lies and violence. Humans are presumed to have such a Machiavellian intelligence to bring out self-interest at the expense of others, mainly because we are part of the biological system. Thus, if the intentions of an agent involve some kind of conflicting goals, we should expect Machiavellian intelligence to be present.

We will here focus on a typical scenario of such an ecosystem where there is one (or more) exploiter agent(s) and one (or more) user agent(s) being exploited. In Fig. 1 we see an example with one exploiter and one user.

The goal for the human exploiter is to make profit from the agent interaction. Besides giving the initial instructions to the exploiter agent, the exploiter most likely has to continually instruct the exploiter agent because of the limited knowledge of the domain of a software agent compared to a human being. The human user and its agent when trying to avert the exploiter agent will perform the same kind of reasoning. The situation will end up in an arms race where the second agent retorts the improvement of the first agent by having its human owner giving it improved instructions. The long-term outcome of a continuing arms race is an improved retort against the unfriendly actions already performed by the opponent, and probably reducing the number of obvious new exploiting behaviours. 
Unlike the traditional descriptions of agent systems based on mental states having belief, desire and intentions [6], we here focus on the humans owners. A human is capable of using knowledge outside the actual domain and arrange it using consciousness. This knowledge is then transferred to the agents through instructions and based on feedback. We believe that this interaction is a key component for understanding the mechanisms behind antagonistic information ecosystems.

\section{Examples of antagonistic information ecosystems}

We will here give some examples of antagonistic conflicts within information ecosystems ranging from an illegal virus attack to legal registrations of users.

\subsection{Computer viruses}

It is easy to imagine an arms race between crackers (the exploiters) and users referring to Fig. 1. In the first phase, the cracker finds out about the weakness of the user's computer system and then creates a program that sends out the viruses, or with the terminology used here, an exploiter agent. To avoid virus-attacks, the user has to use some filtering function or anti-virus program (the user agent). The result may be a refined arms race where the exploiter agent changes the appearance of the virus automatically and the user agent finds these new variants.

Despite its harmful purpose, a virus could be treated as a catalyst for a more robust information ecosystem. We here refer to the biological concept of robustness, i.e., the ability of an agent to properly balance efficiency and efficacy in order to survive in many different environments. The robustness of an ecosystem is then dependent of the robustness of its members. Thus, if ecosystems can be made more robust, they can perform their functions longer and better, which will make costly redesigns unnecessary or at least less costly and less frequent.

A time based security model [8] quantify the exposure time related to a virus attack as dependent on protection, detection and response. These variables in turn have a quality factor involved, expressed as the strength of the mechanism and some active time interval. If the final result of the virus battle is a balance where viruses only in exceptional cases do harm because of an improved time based security, we probably manage better with than without viruses. Just as in natural ecosystem we should not expect systems easy to manipulate. An information ecosystem with humans using their Machiavellian intelligence to control perceiving agents is a much more likely consequence.

\subsection{Spyware}

Today there are millions of Web sites reachable by any Internet user. These users spend more and more time to customize and improve their Web browser 
experiences through third-party browser extensions. These extensions may be able to monitor and report the behavior of the users to anyone needing this information. Cookies are used to register information and later retrieve or modify this information with other Web browsers. A Web site may store information about the user in a cookie that the user does not know about; the cookie mechanism could be considered a form of spyware, abusing the privacy on the Internet.

In general spyware (the exploiter agent) is any technology that aids in gathering information about a person or organization without their knowledge. On the Internet, spyware is programs that are put in someone's computer to secretly gather information about the user and relay it to advertisers or other interested parties. In a recent report, Martin et al. [4] examined business-to-consumer browser add-on products. The research focused on the ways that the browser extensions collected user information, how the data flowed back to the company's database and, how the data capture techniques matched the privacy policies of each company.

"We downloaded 16 Internet Explorer browser extensions and watched them work. A number were well behaved. But others seemed to outridge exploit our hospitality, watching and reporting our every move in the browser, some intercepting data sent to competitors and other reporting back to headquarters on pages we 'securely' downloaded using Secure Socket Layers" [4]

There is a lack of trust against third-party browser extensions and recently antispyware tools (user agents) were offered the Web users. These programs inform the user about any unwanted cookies present and, how to get rid of them.

For both spywares and anti-spywares there is a profit calculation involved. The consumer does not exactly know the purpose of the data collection, but may suspect a third party selling of information. Commercial anti-spyware products replace the first free products. The spyware evolution is similar to the virus example but may be less destructive because:

- The consumer is normally partly informed about the collecting activity (but not about the future use of the data).

- The consumer may be offered an additional web-service or freeware program. The spyware acts as the commercial part of the offered service.

- The data collection may improve the development of e-business.

An arms race within spywares may improve some e-business concepts, i.e. act as a resource generating marketing. Between competing business companies such an improvement may cause advantages against other companies not using spywares.

\section{Conclusions}

Based on the assumption that software agents may mediate the Machiavellian intelligence of their human owners, there are a lot of similarities between 
information and biological ecosystems. The main conclusion we draw from these similarities is that arms race is a major force within information ecosystems.

Both examples presented in section 3 show a development of the information ecosystem through an arms race. From a system perspective, this can be seen as a positive thing because the ecosystem will become more robust. If a user knows about complications caused by exploiting agents and prepare to defend against these intruders, the user will get off better compared to being unprepared. From the user's perspective, the disadvantage is the resources, e.g., money and time, spent on procuring anti-virus and anti-spyware programs.

The Machiavellian intelligence has aroused through an arms race of the capacity to deceive, but this does not mean we lost our (inherited) capability to cooperate. The choice between long-term cooperation and getting some short-term advantage of being selfish is called Prisoner's Dilemma within game theory [7]. Prisoner's dilemma describes the rise of cooperation in both social [1] and natural science [5], within a restricted domain. The results from the analysis of Prisoner's Dilemma can be described as: every agent wins by cooperation, but if everybody else cooperate, the single agent will benefit by being selfish. If no one cooperates everybody will be worse off. Most efforts today to solve this dilemma are done by legislate methods but, as stated previous, we argue that there is a self-adjusting quality that influence the dynamics of antagonistic information ecosystems.

\section{References}

1. Axelrod, R., The Evolution of Cooperation Basic Books Inc. (1984)

2. Dennett, D.C. Darwin's dangerous idea Allen Lane/The Penguin Press, London (1995)

3. Gärdenfors, P. Hur Homo blev sapiens, Bokförlaget Nya Doxa, Nora (2000)

4. Martin Jr, D.M., Smith, R.M., Brittain, M., Fetch, I., and Wu, H., The Privacy Practices of Web Browser Extensions, University of Denver (2000)

5. Maynard Smith, J., Evolution and the theory of games, Cambridge University Press, (1982)

6. Rao, A.S., and Georgeff, M.P., "BDI agents: from theory to practice." In Proceedings of the First International Conference on Multi-Agent Systems, p. 312-319, AAAI Press/The MIT Press (1995)

7. Rapoport, A., and Chammah, A.M., Prisoner's Dilemma A Study in Conflict and Cooperation Ann Arbor, The University of Michigan Press (1965)

8. Scgwartay W. Time Based Security. Practical and Provable Methods to Protect Enterprise and Infrastructure, Networks and Nation Interpact Press (1999)

9. Williams G. C., Adaptation and natural selection Princeton University Press 1966

10. Wilson, E. O., Sociobiology-The abridged edition, Belknap Press (1980) 1975 